\title{
DIAGNÓSTICO ANGIOGRÁFICO DOS TUMORES DE PLEXO CORÓIDE DO VENTRICULO LATERAL
}

\author{
Nélio Garcia de Barros * \\ Gilberto' Machado de Almeida**
}

Em 1960, Donald D. Matson, discutindo um caso apresentado por Faber e Vawter ${ }^{6}$, salientou a importância do papiloma do plexo coróide para diversas especialidades médicas: ao geneticista interessa a possibilidade de o processo ser congênito; ao fisiologista, a razão do hidrocéfalo na ausência de bloqueio ao trânsito do líquido cefalorraqueano (LCR); ao patologista, a relação dêste tumor com os ependimomas e o conceito de malignidade; ao pediatra, a relativa freqüência em tenra idade; ao neurocirurgião, a possibilidade de cura cirúrgica quando precocemente diagnosticado. Entretanto, Matson não salientou o interêsse que tal tumor tem para o neurorradiologista, em vista das dificuldades diagnósticas reconhecidas pela maioria dos autores $4,6,7,10,14$. Realmente, o quadro clínico não apresenta caracteristicas uniformes e o pneumoventriculograma, feito com a quantidade de ar usualmente empregada, é insuficiente, sendo necessário estudo com grande volume de contraste, o que torna o exame muito traumatizante, especialmente em crianças hidrocefálicas.

Em 1955, Ernsting ${ }^{5}$ já sugeria o emprêgo de angiografia cerebral, em conjunto com o estudo pneumográfico, para o diagnóstico dos papilomas do plexo coróide. No caso apresentado por êste autor, o exame, indicado por ter ocorrido hemorragia meníngea, permitiu verificar que o vaso aferente ao tumor era a artéria coróidea anterior. Abbott e col. ${ }^{1}$, em 1957, em outra criança com hemorragia subaracnóidea, realizaram angiografia carotidea bilateral e vertebral, demonstrando que a irrigação do papiloma se fazia pela artéria coróidea posterior. Estes autores salientaram a importância do aspecto angiográfico, caracterizado por pequenas alterações vasculares na região do confluente ventricular. Entretanto, a maioria dos autores com séries grandes dêste tumor limitava-se ao emprêgo das pneumografias, quando a primeira manifestação clínica não consistia em episódio hemorrágico. Em 1958 Pecker e col. ${ }^{12}$ consideraram, como método eletivo para o diagnóstico dos papilomas do plexo coróide, o estudo angiográfico, que tem a vantagem de permitir a avaliação cuidadosa dos pediculos arteriais aferentes. Contudo, trabalhos ulteriores $2,3,4,6,7,9,10,11,14,15$ continuaram a não dar importância às angiografias, havendo apenas referências isoladas 8,13 a casos diagnosticados por êste exame.

Trabalho da Clinica Neurológica da Faculdade de Medicina da Universidade de São Paulo (Prof. Adherbal Tolosa): *Neurorradiologista; ** Neurocirurgião. 
O conceito de malignidade dos tumores do plexo coróide não está relacionado ao aspecto histológico, mas sim com a existência, ou não, de infiltração tumoral. O nosso segundo doente pode ser incluído neste grupo.

\section{MATERIAL}

De 1959 a 1965 foram atendidas, na Clinica Neurológica da Faculdade de Medicina da Universidade de São Paulo, 4 crianças com papiloma e uma com carcinoma do plexo coróide em um dos ventriculos laterais. Os dados relativos à identificação dêsses pacientes e à sintomatologia apresentada figuram no quadro 1 . No quadro 2 figuram os dados obtidos com o EEG e com o exame do LCR. Os pacientes 1 e 2 foram operados, com extirpação total do processo expansivo, estando vivos após 5 anos e 1 ano, respectivamente: no caso 1 a hemiparesia acentuou-se após a operação; o caso 2 não apresenta seqüela. Os casos 3 e 4 faleceram antes de ser realizada a intervenção cirúrgica. O paciente referido no caso 5 faleceu durante o ato operatório.

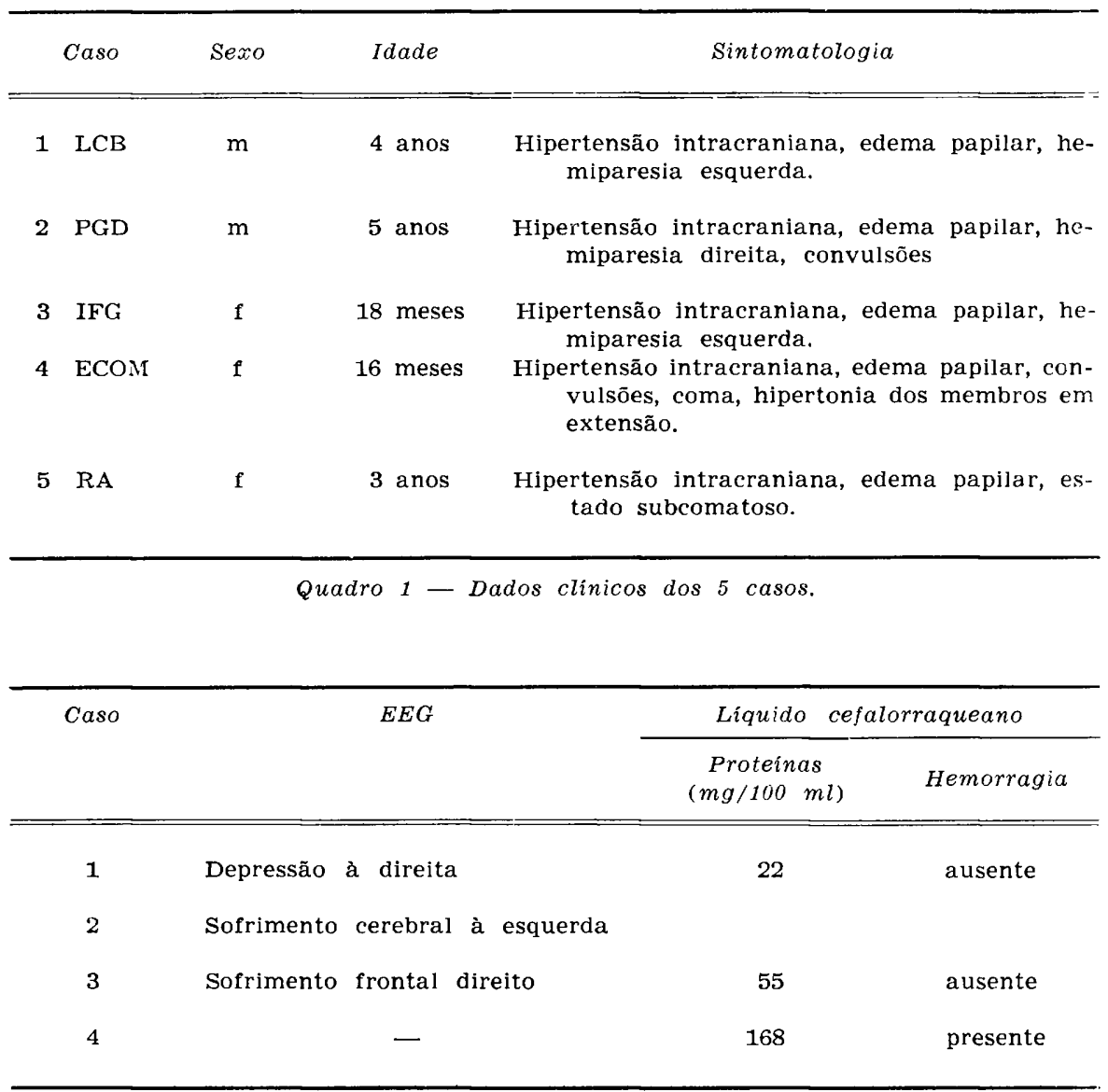

Quadro 2 - Dados de exames auxiliares em 4 casos. 


\section{ESTUDO RADIOLOGICO DOS 5 CASOS}

CASo 1 - LCB (Reg. 558083). Angiografia cerebral via artéria carótida direita. Artéria cerebral anterior estirada e desviada para a esquerda. Artéria cerebral média recalcada para dentro e fortemente elevada, aproximando-se, nos filmes de perfil, da cerebral anterior. A artéria coróidea anterior, muito calibrosa, dirige-se para a área de circulação patológica irregular, medindo três centímetros no maior diâmetro, constituída por fina rêde vascular e situada em projeção parietal, porém abaixo do grupo silviano.

Comentários - O exame sugeriu inicialmente a existência de tumor cistico com pequena massa sólida. No ato cirúrgico veríficamos que o processo expansivo era muito maior, ocupando quase todo o ventrículo lateral dilatado. O grande levantamento das artérias do grupo silviano era causado pela maior dilatação do côrno temporal, bloqueado pelo tumor.

CASo 2 - PGD (Reg. 698908). Angiografia cerebral via artéria carótida esquerda. Desvio para a direita da artéria cerebral anterior, que se apresenta estirada. Artéria cerebral média deslocada para dentro, para a frente e para cima, descrevendo, nos filmes de perfil, trajeto semelhante ao da artéria cerebral anterior. No tempo arterial não houve contrastação da área de circulação patológica, visivel nos tempos capilares e venosos. Os vasos anômalos são pequenos, ocupam área de limites imprecisos, com dois centímetros no maior diâmetro e estão em situação semelhante à do caso anterior. A parte anterior da veia de Galeno encontra-se abaixada. Pneumoventriculografia. Após perfuração parietotemporal esquerda foi atingida cavidade cística contendo líquido fortemente xantocrômico. O ar injetado delimitou cavidade de limites irregulares.

Comentários - Neste caso o estudo pneumográfico nada esclareceu, mostrando apenas cavidade que no ato cirúrgico verificou-se corresponder à porção deformada do ventriculo lateral. O tumor encontrado na operação era muito maior do que a área de circulação patológica observada na angiografia. A acentuada elevação do grupo silviano pôde ser explicada pela maior dilatação do côrno temporal do ventrículo lateral, causada por bloqueio do mesmo.

CASo 3 - IFC (Reg. 718073). Angiografia cerebral via artéria carótida direita. Além dos sinais indiretos de acentuada dilatação ventricular e do levantamento do grupo silviano, nota-se, ao nível do confluente ventricular, área de vascularização exagerada, mais nitida nos tempos venosos, de limites irregulares, medindo cêrca de $4 \mathrm{~cm}$ no maior diâmetro. As artérias coróideas posterior e anterior dirigem-se para os vasos patológicos. Pneumoventriculografia com $50 \mathrm{ml}$ de ar. Deformidade da porção posterior do ventrículo lateral direito, com amputaçāo do côrno occipital.

Comentários - Depois de realizado o estudo pneumográfico, a criança piorou progressivamente, vindo a falecer após 7 dias. $\mathrm{Na}$ necropsia comprovou-se a existência de papiloma do plexo coróide nas porçōes posteriores do ventrículo lateral direito. Neste caso não havia elevação da artéria cerebral média, o que é explicado pelo fato de nâo ter havido bloqueio do côrno temporal.

CASO 4 - ECOM (Reg. 721859). Angiografia cerebral via artéria carótida direita. Não houve contrastação da artéria cerebral anterior; entretanto, todo o sistema vertebrobasilar foi visibilizado. Ao nivel do confluente ventricular do lado esquerdo há grande área arredondada de circulação patológica, mais nitida nos 
tempos venosos, medindo cêrca de $6,5 \mathrm{~cm}$ no maior diâmetro e de limites nítidos. A irrigação do processo expansivo é feita principalmente às custas da artéria coróidea posterior esquerda. Angiografia cerebral via artéria carótida esquerda. Além. da visibilização mais nitida do processo expansivo demonstrado no exame anterior, notam-se sinais indiretos de dilatação ventricular, sem desvio da cerebral anterior, mas elevacão do grupo silviano (fig. 1).

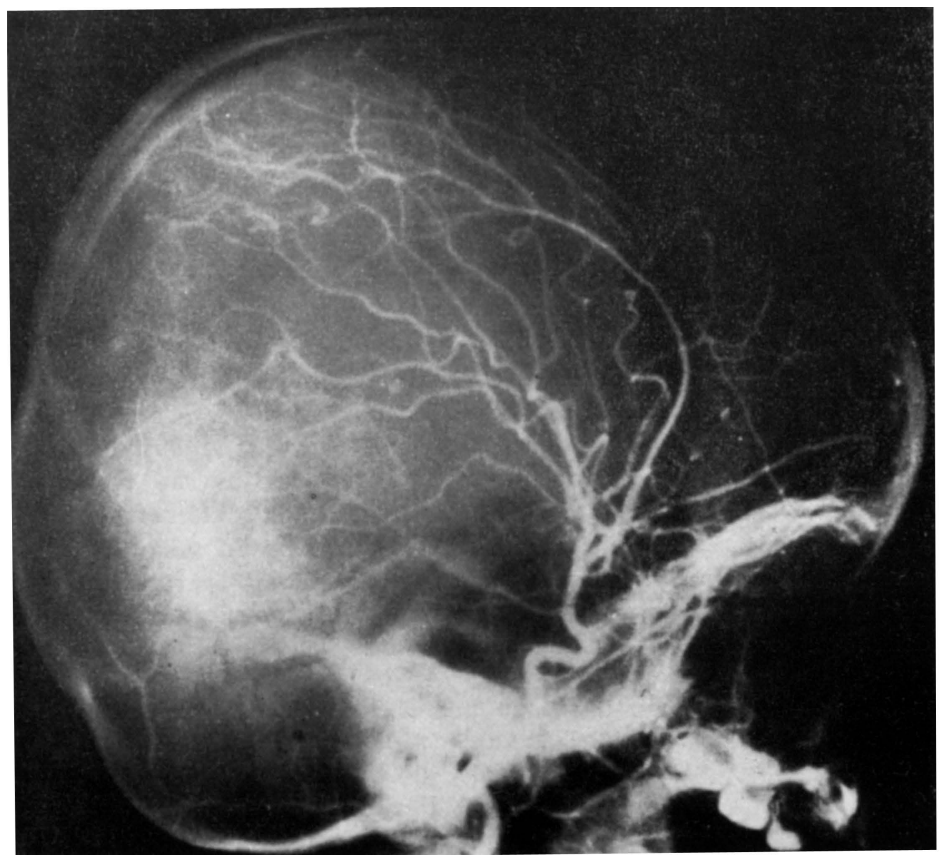

Fig. 1 - Caso 4 (ECOM) - Angiografia cerebral mostrando a área de circulação patológica, os sinais indiretos de dilataçuo ventricular e a elevação do grupo silviano. Papiloma.

Comentários - O exame foi feito com a criança em estado muito grave, devido à hemorragia meningea maciça e à grande hipertensāo intracraniana. Após a angiografia cerebral a paciente continuou a piorar, apesar da drenagem ventricular contínua, vindo a falecer no dia seguinte. Êste foi o único dos nossos casos em que a área de circulação patológica demonstrada nas angiografias correspondeu ao volume real do processo expansivo, comprovado na necropsia.

CASo 5 - RA (Reg. 773214). Angiografia cerebral via artéria carótida direita. Estiramento e desvio da artéria cerebral anterior para a esquerda. Artéria cerebral média estirada e elevada. Área de circulação patológica volumosa medindo, nos filmes de perfil, $9,5 \times 6,5 \mathrm{~cm}$, situada na região parietal. A artérı́a coróidea anterior, ramos da cerebral anterior e da cerebral média dirigem-se para o tumor, onde notamos a presença de numerosos vasos neoformados, fístulas arteriovenosas e retenção de contraste (fig. 2). 


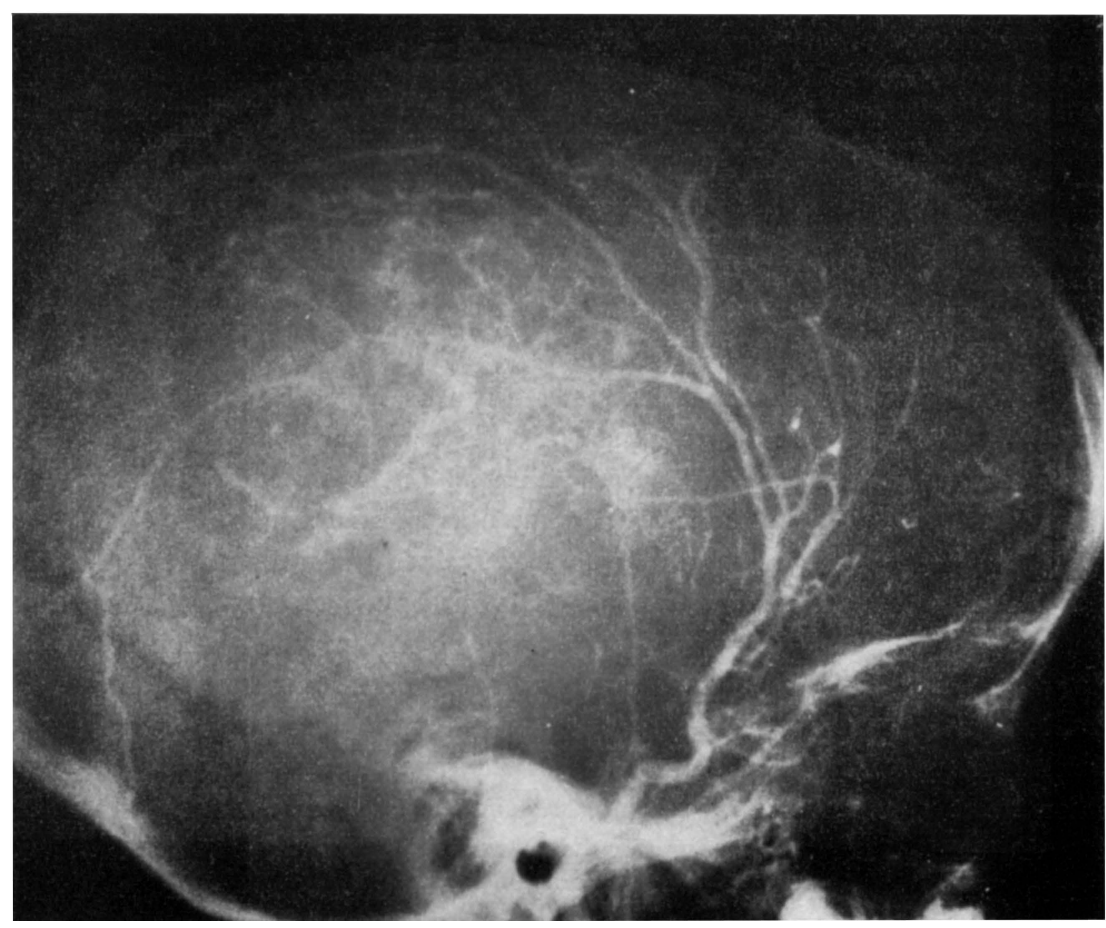

Fig. 2 - Caso 5 (RA) - Angiografia cerebral mostrando grande área de circulacio patológica, retencio de contraste, vasos neoformados, fístula arteriovenosa, estiramento dos vasos e elevacio do grupo silviano. Carcinoma.

Comentários - o quadro angiográfico difere dos anteriores. Os caracteres de malignidade são evidentes, a área de circulação patológica com retenção de contraste é maior que nos outros casos. Esta criança chegou ao hospital em estado grave, tendo sido operada no mesmo dia. Desde o inicio do ato cirúrgico o edema cerebral era muito acentuado e o sangramento tão abundante que levou a paciente a paciente ao choque e ao óbito. A biopsia revelou tratar-se de carcinoma, invasivo, do plexo coróide.

\section{DISCUSSAO}

Os papilomas do plexo coróide determinam quadros clínicos diversos. A hipertensão intracraniana é freqüente, podendo estar, ou não, acompanhada de sinais de localização. Outras vêzes, o quadro inicia-se por hemorragia meníngea, como ocorreu em nosso caso 4 . Nos pacientes dêste último grupo o exame neurorradiológico que tem sido empregado com mais freqüência é a angiografia cerebral. Nos demais casos, em que o processo expansivo acarreta quadro clínico de hipertensão intracraniana, a maioria dos autores opta pelas pneumografias. Acreditamos que a experiência adquirida com alguns casos referidos na literatura $1,5,8,1 \cdots, 13$ e os 5 agora rela- 
tados atestam as vantagens da angiografia cerebral. Este exame é menos traumatizante do que as pneumografias realizadas com a quantidade de contraste necessária para o diagnóstico dos papilomas do plexo coróide dos ventrículos laterais. Em dois de nossos doentes foram feitas ventriculografias. No caso 2 o exame mostrou um cisto, não dando maiores informaçōes quanto à etiologia do processo. No caso 3 a pneumoventriculografia mostrou deformidade da porção posterior do ventriculo lateral; só a análise em conjunto com a angiografia permitiu firmar o diagnóstico radiológico de papiloma do plexo coróide.

As angiografias demonstraram, em todos os casos de papilomas, a existência de área de circulação patológica, mais nítida nos tempos venosos. Em 3 doentes (casos 1, 2 e 3) havia delicada rêde vascular e em um caso (4) os vasos patológicos formavam grande conglomerado, mostrando nìtidamente o volume do processo expansivo. É interessante chamar a atenção para o fato de que só neste último enfêrmo havia correspondência entre o tamanho da área de circulação patológica e o volume do tumor. O quadro histopatológico dêste caso foi semelhante ao encontrado nos demais, sem caracteres de malignidade. A angiografia do caso 5 apresentou aspecto diferente. A pres€nça de fístulas arteriovenosas, o grande número de vasos neoformados e a extensa área de retenção de contraste sugeriam malignidade do tumor. A biopsia revelou tratar-se de carcinoma, pois, além do aspecto histológico indicar malignidade, havia infiltração de áreas vizinhas, fato que não ocorreu em nenhum dos outros 4 casos.

Em 4 dos doentes ( $\operatorname{casos} 1,2,4$ e 5) havia desvio, para dentro e para cima, dos vasos do grupo silviano. Nos casos 1,2 e 5 êste deslocamento arterial era tão acentuado que não poderia ser explicado pela localização do tumor. Realmente, a maior dilatação do côrno temporal, por bloqueio do mesmo, foi a causa do grande desvio arterial, fato êste que constitui mais um elemento para se suspeitar do diagnóstico de papiloma do plexo coróide.

Em todos os pacientes havia sinais indiretos de dilatação ventricular e, em três casos (1, 2 e 5), desvio contralateral da artéria cerebral anterior.

Outra vantagem do exame angiográfico é permitir o estudo dos principais vasos aferentes e eferentes ao tumor, o que pode ter importância na orientação da tática cirúrgica.

Em lactentes com quadro clínico de hidrocefalia muitos cirurgiões realizam a ventrículo-auriculostomia, sem estudos neurorradiológicos ou apenas com ventriculografias insuficientes. Desta maneira podem ter passado despercebidos vários casos de papilomas do plexo coróide. Parece-nos interessante relembrar que vários elementos clínicos e laboratoriais fazem suspeitar da existência de tal tumor, obrigando a exploração neurorradiológica completa. Entre êstes dados têm importância o início relativamente tardio do quadro clínico, a existência de papiledema, as alterações eletrencefalográficas, a xantocromia do líquido cefalorraqueano e, principalmente, a hiperproteinorraquia. 


\section{RESUMO}

Os autores consideram a angiografia cerebral como método de escolha para o diagnóstico dos tumores de plexo coróide dos ventrículos laterais. As pneumografias, por necessitarem de grande quantidade de contraste, são muito traumatizantes e, não raro, insuficientes. Nos 5 casos apresentados, as angiografias cerebrais demonstraram a existência de circulação patológica, permitindo o diagnóstico correto. Outro elemento interessante é a demonstração da dilatação do côrno temporal bloqueado. Além disso, o exame permite estudar os vasos aferentes e eferentes, facilitando, desta maneira, a tática cirúrgica. O aspecto angiográfico de um caso de carcinoma foi diverso dos 4 casos de papiloma.

SUMMARY

Angiographic diagnosis of tumors of the choroid plexus of the lateral ventricles

The authors believe that cerebral angiography is the best neuroradiologic procedure for the diagnosis of tumors of the choroid plexus of the lateral ventricles. Pneumographs needing a large quantity of contrast material are very traumatic and occasionally insufficient. In the 3 cases reported cerebral angiograms disclosed the presence of pathological circulation, enhancing a correct diagnosis. Another interesting point is the demonstration of the dilatation of the blocked temporal horn. In addition, angiography allows the study of the afferent and efferent vessels, thus facilitating surgical procedures. The angiographic picture in a case of carcinoma was different from those found in four papillomata cases.

\section{REFERENCIAS}

1. ABBotT, K. H.; ROLlas, Z. H. \& MEAGHER, J. N. - Choroid plexus papilloma causing spontaneous subarachnoid hemorrhage. J. Neurosurg. 14:566-570, 1957. 2. BAAR, H, S. \& GALINDO, J. - Bilateral papilloma of the choroid plexus. J. Maine med. Ass. 55:95-99, 1964. 3. BOHM, E. \& STRANG, R. - Choroid plexus papillomas. J. Neurosurg. 18:493-500, 1961. 4. CROFTON, F. D. L. \& MATSON, D. D. - Roentgenologic study of choroid plexus papillomas in childhood. Amer. J. Roentgenol. 84:479-487, 1960. 5. ERNSTING, J. - Choroid plexus papilloma causing spontaneous subarachnoid hemorrhage. J. Neurol. Neurosurg. Psychiat. 18:134136, 1955. 6. FABER, S. \& VAWTER, G. F. - Clinical Pathological Conference. J. Pediat. 56:834-838, 1960. 7. FAIRBURN, B. - Choroid plexus papilloma and its relation to hydrocephalus. J. Neurosurg. 17:166-171, 1960. 8. HAMMON, W. M. \& KEMPE, L. G. - Angiographic appearance of a papilloma of the choroid plexus of the lateral ventricle. J. Neurosurg. 20:711-714, 1963. 9. HILLIER Jr., W. F. Intraventricular papilloma. Report of a case with successful removal. W.Va med. J. 59:341-344, 1963. 10. LAURENCE, K. M.; HOARE, R. D. \& TILL, K. - The diagnosis of the choroid plexus papilloma of the lateral ventricle. Brain 84:628641, 1961. 11. MATSON, D. D. \& CROFTON, F. D. L. - Papilloma of the choroid 
plexus in childhood. J. Neurosurg. 17:1002-1027, 1960. 12. PECKER, J.; BERDET, H. \& MESSIMY, R. - Les papillomes des plexus choroides des ventricules latéraux. Etude clinique et anatomique de 5 cas operés. Neuro-chirurgie 4:45-60, 1958.13. SEARS, R. A. \& BURNETT, S. W. - Angiographic appearance of a papilloma of the choroid plexus. J. Neurosurg. 18:822-825, 1961. 14. SJOGREN, I.; GROTTE, G. \& OLDING, L. - Choroid plexus papilloma and infantile hydrocephalus. Acta paediat. 53:182-187, 1964. 15. WILKINS, H. \& RUTLEDGE, B. J. - Papillomas of the choroid plexus. J. Neurosurg. 18:14-18, 1961.

Clinica Neurológica - Faculdade de Medicina da Universidade de São Paulo - Caixa Postal 3461 - Sío Paulo, SP - Brasil. 